\title{
MODELO DE EFEITOS FIXOS COM MEDIDA REPETIDA APLICADO EM EXPERIMENTOS DE MELHORAMENTO GENÉTICO DO CUPUAÇUZEIRO ${ }^{1}$
}

\author{
RAFAEL MOYSÉS ALVES ${ }^{2}$, MARIA REGINA MADRUGA³ ${ }^{3}$ HÉLITON RIBEIRO TAVARES ${ }^{4}$, \\ TARCÍSIO DA COSTA LOBATO ${ }^{5}$, TEREZINHA FERREIRA DE OLIVEIRA ${ }^{6}$
}

RESUMO- Esta pesquisa foi desenvolvida para avaliar o desempenho vegetativo de progênies de cupuaçuzeiro, na fase de imaturidade, por meio da análise de medidas repetidas no tempo. Aárea experimental foi instalada no município de Tomé-Açu, Nordeste do Estado do Pará. Foram empregadas 25 progênies de irmãos completos de cupuaçuzeiro, que tiveram o desenvolvimento vegetativo (altura e diâmetro da planta) monitorado durante três anos. Por conta da natureza longitudinal das observações, foi avaliado primeiramente, por meio do teste de esfericidade de Mauchly, qual o tipo de análise estatística deveria ser aplicada. Para a variável diâmetro, a condição de esfericidade não foi violada, sendo assim, procedeu-se à análise no esquema de delineamento de parcelas subdivididas no tempo. Para a variável altura, o teste foi significativo, indicando necessariamente avaliar qual a melhor estrutura que explique a correlação dos erros, sendo escolhida a estrutura de covariâncias Simetria Composta Heterogênea. Foram encontradas diferenças entre as progênies somente no terceiro ano de avaliação, sendo que a variável diâmetro da planta permitiu discriminar melhor as progênies avaliadas.

Termos para indexação: Theobroma grandiflorum, estruturas de covariâncias, progênies.

\section{FIXED EFFECT MODELS WITH REPEATED MEASURES APPLIED TO GENETICS IMPROVEMENT OF CUPUASSU TREE}

\begin{abstract}
This study was conducted to evaluate vegetative performance of cupuassu progenies, in the immaturity phase, through analysis of repeated measures. The experimental area was installed in the municipality of Tomé Açu, Northeast of the state of Para. We employed 25 full-sib progeny of cupuassu, who had vegetative growth (height and diameter of the plant), monitored for three years. Because of the nature of longitudinal observations it was assessed primarily through Mauchly sphericity test, which type of statistical analysis should be applied. For the variable diameter, sphericity condition was not violated, therefore, proceeded to review the design scheme of split plot. For the height variable, the test was significant, indicating necessarily evaluate the best structure to explain the correlation of errors, being chosen the covariance structure Heterogeneous Composed Symmetry. Differences were found between the progenies only in the third year of evaluation, and the variable diameter of the plant allowed better discriminate the progenies.

Index terms: Theobroma grandiflorum, covariance structures progenies.
\end{abstract}

\footnotetext{
1(Trabalho 234-14). Recebido em: 02-09-2014. Aceito para publicação em: 04-08-2015.

${ }^{2}$ Pesquisador, Embrapa Amazônia Oriental, CP 48, Belém-PA. E mail: rafael-moyses.alves@embrapa.br

${ }^{3}$ Professora da Faculdade de Estatística, UFPA, Belém - PA. E mail: madruga@ufpa.br

${ }^{4}$ Professor da Faculdade de Estatística, UFPA, Belém - PA.E mail: heliton@ufpa.br

${ }^{5}$ Mestrando em Estatística, UFPA, Belém - PA. E mail: tarcisiolobato@yahoo.com.br

${ }^{6}$ Professora da Faculdade de Estatística, UFPA, Belém - PA.E mail: tfo@ufpa.br
} 


\section{INTRODUÇÃO}

O cupuaçuzeiro, Theobroma grandiflorum (Willd. ex Spreng.) Schum., é uma frutífera que hoje se encontra dispersa por toda a Bacia Amazônica, sendo a Amazônia brasileira a única reserva de variabilidade genética nativa da espécie (ALVES et al., 2013).

$\mathrm{O}$ fruto é exótico e muito ácido $(\mathrm{pH} \cong$ $3.2)$, com baixo teor de açúcar $\left(\mathrm{Brix} \cong 11^{\circ}\right)$ e com aroma muito forte. Sua polpa tem excelente sabor e potencial agroeconômico elevado, pois é utilizada para preparar um produto similar ao chocolate, assim como na fabricação de geleias, sucos, sorvetes, doces, iogurtes, manteiga e ingredientes cosméticos (OLIVEIRA; GENOVESE, 2013).

O cultivo dessa espécie teve início na década de 1970. Até essa época, a produção de frutos de cupuaçuzeiro provinha, basicamente, das populações nativas existentes no sudeste do Pará e noroeste do Maranhão, regiões estas tidas como o centro de origem e diversidade do cupuaçuzeiro (ALVES et al., 2013). Atualmente, cerca de 30.000 ha encontram-se implantados na região amazônica, sendo 13.000 ha no Estado do Pará, onde o município de Tomé-Açu, com 3.000 ha, é o mais importante (SAGRI, 2015).

Para a realização dos plantios pioneiros, os produtores utilizaram sementes sem nenhum critério de seleção. Após alguns anos de cultivo, a doença conhecida como vassoura-de-bruxa, cujo agente etiológico é o Moniliophthora perniciosa (Stahel) Singer, passou a atacar os plantios, inicialmente de maneira endêmica e, posteriormente, na forma de epidemia (ALVES et al., 2009).

Para viabilizar o cultivo, dessa fruteira, a Embrapa Amazônia Oriental estruturou um programa de melhoramento genético do cupuaçuzeiro, que visava desenvolver variedades mais produtivas e resistentes a doenças (ALVES et al., 2010).

Em 2002, foram lançadas as primeiras cultivares de cupuaçuzeiro, que tinham como principal característica a tolerância à vassoura-debruxa, além de boa produção de frutos (ALVES et al., 2010). Paralelamente, o programa de melhoramento buscou um material mais produtivo, propagado por sementes, e que apresentasse fontes distintas de resistência à vassoura-de-bruxa. A necessidade de diversificação das fontes de resistência é decorrente do risco que correria o produtor de utilizar materiais uniformes. Em 2012, foi lançada a BRS Carimbó, que apresentava características de boa produção de polpa e sementes, aliada a boa resistência a essa doença (ALVES; FERREIRA, 2012).

Novos genótipos, ainda mais produtivos e resistentes aos patógenos do cupuaçuzeiro, fazem-se necessários, para tornar a atividade produtiva ainda mais segura. Entretanto, o custo experimental é muito elevado, pois cada planta deverá ser testada em $25 \mathrm{~m}^{2}$ de terreno, por um período mínimo de dez anos. Daí a necessidade de identificar caracteres vegetativos que possibilitem correlacioná-los com características produtivas ou, pelo menos, descartar os indivíduos que não teriam bom desempenho na fase produtiva.

Muitos são os campos de estudo onde se efetuam várias observações sobre a mesma unidade experimental ao longo do tempo. Planejamentos do tipo longitudinais são frequentemente utilizados em diversas áreas de pesquisa, porque permitem avaliar mudanças globais ou individuais ao longo do tempo (MIRANDA et al., 2009; ROSA et al., 2013).

Como as medidas são repetidas de modo sistemático, nas mesmas unidades experimentais, espera-se que exista uma correlação não nula entre as medidas e uma heterocedasticidade das variâncias nas diversas ocasiões (CECON et al., 2008).

Devido à facilidade na análise e à interpretação dos resultados, experimentos em parcelas subdivididas no tempo têm sido frequentemente utilizados na análise de dados com medidas repetidas. No entanto, para que os resultados referentes à subparcela tempo e à interação tempo $x$ tratamento sejam válidos, é necessário que as pressuposições da análise usual sejam atendidas, ou seja, que os erros da subparcela apresentem distribuição normal, sejam independentes e identicamente distribuídos e com variâncias constantes (FREITAS et al., 2008).

$\mathrm{Na}$ verificação da hipótese de erros homocedásticos, uma condição necessária e suficiente para que o teste F, referente à subparcela, seja válido, é especificar uma matriz de covariâncias com as variâncias da diferença entre pares de erros todas iguais. Essa condição foi descrita por Huynh e Feldt (1970) e denominada HUYNH-FELDT (HF), sendo a condição de simetria composta por um caso particular (XAVIER; DIAS, 2001; FREITAS et al., 2008).

Para verificar a condição H-F, foi proposto o teste de esfericidade de Mauchly (1940), que consiste em verificar se uma população normal multivariada apresenta variâncias iguais e correlações nulas (XAVIER; DIAS, 2001). Caso seja aceita a condição de esfericidade, pode-se continuar com a análise no esquema de parcelas subdivididas sem que os resultados baseados no teste $\mathrm{F}$ fiquem comprometidos (FREITAS et al., 2008).

No entanto, se a condição de esfericidade não for satisfeita, ou seja, se o teste de Mauchly for significativo, uma alternativa é realizar a correção 
para os graus de liberdade do teste $\mathrm{F}$ para os fatores da subparcela. Outra alternativa é considerar a análise de variância multivariada (MANOVA) em que nenhuma restrição é feita quanto à estrutura de variâncias e covariâncias, ou ainda considerar o ajuste de modelos mistos, em que diversas estruturas de covariâncias são testadas, selecionando aquela que melhor explique o comportamento dos dados (XAVIER; DIAS, 2001).

O objetivo deste trabalho foi avaliar o desenvolvimento vegetativo de 25 progênies de cupuaçuzeiro, por meio da análise de medidas repetidas, verificando, por meio do teste de esfericidade, qual tipo de análise estatística é mais adequada (parcelas subdivididas no tempo ou modelos mistos), além de verificar se ocorrem efeitos de progênie, tempo e a interação progênie x tempo.

\section{MATERIAIS E MÉTODOS}

Aárea experimental foi instalada no município de Tomé-Açu, nordeste do Estado do Pará - Brasil, nas coordenadas geográficas de $02^{\circ} 33^{\prime} 0.5^{\prime \prime} \mathrm{S}$ e $48^{\circ}$ $15^{\prime} 47.3^{\prime \prime} \mathrm{W}$.

O clima da região é quente e úmido, enquadrando-se ao tipo climático Ami, da classificação de Köppen, com temperatura média de $26,4^{\circ} \mathrm{C}$, máxima de $32,8^{\circ} \mathrm{C}$ e mínima de $21,9^{\circ} \mathrm{C}$, com precipitação pluviométrica média anual de 2.300 mm. O solo é do tipo Latossolo Amarelo distrófico, profundo, de textura média.

O experimento foi constituído por 25 progênies de irmãos completos de cupuaçuzeiro. Tais progênies têm estrutura genética de um tricomposto, sendo inicialmente produzidos híbridos intraespecíficos, cujas progênies foram avaliadas em campo.

As melhores progênies e as melhores plantas dentro de cada progênie foram selecionadas e depois cruzadas com um clone diferente dos parentais, gerando as progênies tricompostas que formaram o material da presente pesquisa. Essas progênies foram codificadas por números de três algarismos. As cultivares Coari (174), Codajás (186), Manacapuru (215) e Belém (286) foram os principais parentais dessas progênies, além dos clones 434;513; 554; 1.074 e Sekó.

Foi empregado o delineamento experimental de blocos casualizados, com 25 tratamentos, cinco repetições e três plantas por parcela. As variáveis respostas utilizadas foram a altura e o diâmetro da planta, avaliadas em junho/2008, julho/2009 e setembro/2010.

Para um experimento com delineamento de parcelas subdivididas, com medidas repetidas no tempo, foi utilizado o seguinte modelo (VONESH; CHINCHILLI, 1997)

$$
y_{\mathrm{jk}}=\mu+\beta_{i}+\tau_{\mathrm{j}}+\gamma_{\mathrm{k}}+(\tau \gamma)_{\mathrm{jk}}+\mathrm{e}_{\mathrm{ijk}},
$$

em que, $Y_{i j k}$ é o valor observado para a variável resposta (altura ou diâmetro da planta de cupuaçuzeiro) no $k$-ésimo tempo para a j-ésima progênie no $i$-ésimo bloco; $\mu$ é uma constante comum a todas as observações; $\beta_{i}$ é o efeito do $i$-ésimo bloco; $\tau_{j}$ é o efeito da j-ésima progênie; $\gamma_{\kappa}$ é o efeito do $k$-ésimo tempo observado; $(\tau \gamma)_{j k}$ é o efeito da interação entre a $j$-ésima progênie e o $k$-ésimo tempo, $\mathrm{e}_{e i j k}$ é o erro aleatório, com distribuição normal e matriz de covariância $\Sigma$, associado à observação do $k$-ésimo tempo para a $j$-ésima progênie no $i$-ésimo bloco (variação do acaso sobre as observações), com $i=1,2,3,4,5 ; j=1, \ldots, 25$ e $k=1,2,3$.

A fim de verificar se uma população possui variâncias iguais e correlações nulas, aplicou-se o teste de esfericidade de Mauchly (1940), em que a hipótese nula foi rejeitada ao nível $\alpha=0,05$ de significância, quando:

$$
\begin{gathered}
-\gamma \operatorname{In} W>X^{2} \alpha \mathrm{f}, \text { em que, } \\
W=\frac{(t-1)^{t-1}\left|\csc ^{\prime}\right|}{\left(\operatorname{tr}\left(\csc ^{\prime}\right)\right)^{t-1}} \text { e } \gamma=(g b-g-b+1)-\frac{2 t^{2}-3 t+3}{6(t-1)},
\end{gathered}
$$

em que, $t$ é o número de tempos avaliados; $S$ é a matriz de covariâncias amostrais para o erro da subparcela, com $(g b-g-b+1)$ graus de liberdade; $C$ é a matriz de coeficientes dos contrastes ortogonais normalizados, que sob $\mathrm{H}_{0}$ possui distribuição assintótica qui-quadrada com $f=0,5 * t(t-1)-1$ graus de liberdade; $b$ é o número de blocos, e $g$ é o número de tratamentos (XAVIER; DIAS, 2001; LYRA et al., 2006; FREITAS et al., 2008).

Para o caso em que a condição de esfericidade foi aceita, utilizou-se da análise usual de parcelas subdivididas no tempo, em que foram testadas as hipóteses de não existência do efeito da interação Progênie $x$ Tempo, efeito de Tempo e efeito de Progênie. As médias dos efeitos significativos foram agrupadas por meio do procedimento Scott-Knott, ao nível de $5 \%$ de probabilidade. Para essa análise, foi utilizado o pacote ExpDes (FERREIRA et al., 2013) do software livre R (R Core Team, 2015).

No caso em que a condição de esfericidade não foi aceita, utilizou-se da análise de modelos mistos, em que foram avaliadas quatro estruturas de covariâncias $(\Sigma)$ que modelam a dependência dos erros do modelo (1): não estruturada (UN), simetria 
composta (CS), simetria composta heterogênea (CSH) e autorregressiva de ordem 1 (AR1). A estrutura dessas matrizes é apresentada na Tabela 1. Para selecionar a estrutura de covariâncias que melhor explique a correlação residual, foram utilizados os Critérios de Informação de Akaike (AIC) e o Critério Bayesiano de Schwarz (BIC), sendo escolhido o modelo com menor valor de AIC e BIC (CECON et al., 2008; CESTARI et al., 2012; SILVA et al., 2015).

Para efetuar o ajuste do modelo e selecionar a melhor estrutura de covariâncias, foi utilizado o software SAS (SAS INSTITUTE, 2012) por meio do procedimento PROC MIXED e método da máxima verossimilhança restrita. Da mesma forma que na análise usual, foram testadas as hipóteses dos efeitos fixos, sendo as médias de altura e de diâmetro da planta de cupuaçuzeiro agrupadas dentro de cada ano, por meio do procedimento Scott-Knott, ao nível de $5 \%$ de probabilidade, utilizando o erro-padrão gerado pela análise de medidas repetidas no SAS (CECON et al., 2008). Para essa análise, foi utilizado o pacote ScottKnott (JELIHOVSCHI et al., 2014) do software livre R (R Core Team, 2015).

\section{RESULTADOS E DISCUSSÃO}

Na Tabela 2, são apresentados os resultados do teste de esfericidade para as variáveis altura e diâmetro da planta de progênies de cupuaçuzeiro em Tomé-Açu, Pará. Observa-se que, para a variável altura, o teste foi significativo (valor de qui-quadrado aproximado igual a 18,08 ) ao nível de $5 \%$, violando assim a suposição de esfericidade, ou seja, a matriz de covariâncias não pode ser considerada do tipo Huynh-Feldt (HF). Faz-se necessário, então, avaliar qual a melhor estrutura de covariâncias que explique a dependência entre os erros do modelo. Já para a variável diâmetro da planta, o teste de Mauchly foi não significativo (valor de qui-quadrado aproximado igual a 5,94), não rejeitando a hipótese de matriz de covariâncias do tipo H-F; logo, os resultados da análise de variância no esquema de delineamento de parcelas subdivididas no tempo são válidos. Assim, procedeu-se à análise, sendo as médias agrupadas pelo procedimento de Scott-Knott a 5\%.

Lyra et al. (2006), avaliando 12 parâmetros físico-químicos de amostras de água coletadas no lençol freático de um plantio de cana-de-açúcar, em 3 ocasiões, verificaram que em somente 3 variáveis a condição de esfericidade não foi rejeitada, indicando assim existir uma dependência entre as mensurações ao longo do tempo. Os autores também comentam que o teste de esfericidade deve ser efetuado antes de decidir qual análise considerar, se a de medidas repetidas ou a de parcelas subdivididas.

Ambrosano et al. (2011) avaliaram a variável $\mathrm{TCH}$ (Tonelada de Cana por Hectare) em quatro anos consecutivos e verificaram que a matriz de covariância atendia à condição $\mathrm{H}-\mathrm{F}$, sendo feita a análise de parcelas subdivididas no tempo. Já Freitas et al. (2008), analisando a mesma variável em três anos consecutivos, verificaram que a condição de esfericidade foi violada, sendo feita a correção para os graus de liberdade referentes à subparcela. Os autores também comentam que, além da facilidade de aplicação e interpretação, a análise de parcelas subdivididas no tempo é uma alternativa eficiente, desde que a condição de esfericidade seja atendida.

Para a variável altura da planta, foi selecionada a estrutura de covariâncias Simetria Composta Heterogênea, pois apresentou menor valor de AIC e BIC dentre as quatro estruturas avaliadas (Tabela 3 ). Essa mesma estrutura de covariâncias foi selecionada no estudo desenvolvido por Cecon et al. (2008) ao avaliarem 50 clones de café "Conilon" por meio da análise de medidas repetidas. Silva et al. (2015), ao avaliarem nove estruturas de covariâncias em quatro experimentos de cana-de-açúcar, entre os anos de 2004 a 2009, concluíram que as estruturas aqui avaliadas (UN e CSH) se destacaram dentre as demais, considerando os genótipos aleatórios.

Na Tabela 4, são apresentados os resultados dos testes dos efeitos fixos do modelo considerando a análise de parcelas subdivididas para a variável altura da planta e o modelo misto com matriz de covariâncias dos erros Simetria Composta Heterogênea para a variável diâmetro da planta. Os resultados indicam que, para a variável altura da planta, os efeitos da interação progênie $x$ tempo, progênie e tempo foram todos significativos $(\mathrm{p}<0,05)$. Já para a variável diâmetro da planta, somente o efeito de progênie não foi significativo. A interação e o efeito de tempo significativos indicam que ocorre um crescimento das distâncias médias em proporções diferentes, ao longo do tempo, para ambas as variáveis analisadas. No diâmetro de plantas de cupuaçuzeiro, o efeito não significativo de progênie indica que as mesmas apresentaram uma igualdade das distâncias médias, o que não ocorreu para a altura da planta, em que as progênies se diferenciaram.

$\mathrm{O}$ agrupamento das médias, por meio do procedimento Scott-Knott para as variáveis altura e diâmetro da planta, em cada ano, é apresentado na Tabela 5. Observa-se que, para ambas as variáveis analisadas, não houve diferença entre as médias das progênies nos dois primeiros anos, mostrando que o crescimento inicial das plantas foi uniforme. 
Resultados semelhantes foram obtidos por Pasa et al. (2014) ao avaliarem mudas de mirtileiro, em que não encontraram diferenças significativas entre as cultivares estudadas para a variável altura da planta no primeiro ano de avaliação. Ao avaliarem a altura da planta de dois clones de eucalipto, por meio da análise de medidas repetidas, Aparício et al. (2010) também não encontraram diferenças significativas entre os tratamentos de limpeza nos primeiros quatros meses de avaliação.

No terceiro ano de avaliação, em que ocorreram diferenças entre as progênies, observouse que, para ambas as variáveis analisadas, foram formados dois grupos de progênies pelo procedimento de Scott-Knott. A variável diâmetro da planta discriminou melhor as progênies, em que o grupo com as maiores médias foi formado por oito progênies, enquanto para a variável altura da planta, 14 progênies foram agrupadas com as maiores médias. As vantagens da variável diâmetro sobre altura da planta, para seleção de progênies de cupuaçuzeiro, já tinham sido apontadas em pesquisas anteriores (SOUZA et al., 1998).

A progênie 129 destaca-se com maior média de altura e diâmetro da planta $(2,77 \mathrm{~cm}$ e $7,25 \mathrm{~cm}$, respectivamente). As progênies 118; 122; $127 ; 131 ; 136 ; 138$ e 160 também se destacaram quanto à variável diâmetro. Destas oito progênies, sete tiveram como parentais os clones 174 e 186 , indicando serem genótipos que conferem bom desenvolvimento vegetativo aos seus descendentes, além de transmitirem genes de resistência à vassourade- bruxa (ALVES et al., 2010). A progênie 133 apresentou o mesmo valor de média de altura que a progênie 129, mas em relação ao diâmetro, foi classificada no grupo de menores médias, com valor de $6,56 \mathrm{~cm}$.

TABELA 1 - Estruturas de matrizes de covariâncias utilizadas neste trabalho e implementadas no software SAS.

Nome

Não Estruturada (UN)

Simetria Composta (CS)

Simetria Composta Heterogênea (CSH) $\Sigma=\left[\begin{array}{cccc}\sigma_{1}^{2} & \sigma_{1} \sigma_{2} \rho & \sigma_{1} \sigma_{3} \rho & \sigma_{1} \sigma_{4} \rho \\ \sigma_{2} \sigma_{1} \rho & \sigma_{2}^{2} & \sigma_{2} \sigma_{3} \rho & \sigma_{2} \sigma_{4} \rho \\ \sigma_{3} \sigma_{1} \rho & \sigma_{3} \sigma_{2} \rho & \sigma_{3}^{2} & \sigma_{3} \sigma_{4} \rho \\ \sigma_{4} \sigma_{1} \rho & \sigma_{4} \sigma_{2} \rho & \sigma_{4} \sigma_{3} \rho & \sigma_{4}^{2}\end{array}\right]$

Autorregressiva de Ordem 1 (AR1)
Estrutura

$$
\begin{gathered}
\Sigma=\left[\begin{array}{llll}
\sigma_{1}^{2} & \sigma_{21} & \sigma_{31} & \sigma_{41} \\
\sigma_{21} & \sigma_{2}^{2} & \sigma_{32} & \sigma_{42} \\
\sigma_{31} & \sigma_{32} & \sigma_{3}^{2} & \sigma_{43} \\
\sigma_{41} & \sigma_{42} & \sigma_{43} & \sigma_{4}^{2}
\end{array}\right] \\
\Sigma=\left[\begin{array}{llll}
\sigma^{2} & \sigma_{1} & \sigma_{1} & \sigma_{1} \\
\sigma_{1} & \sigma^{2} & \sigma_{1} & \sigma_{1} \\
\sigma_{1} & \sigma_{1} & \sigma^{2} & \sigma_{1} \\
\sigma_{1} & \sigma_{1} & \sigma_{1} & \sigma^{2}
\end{array}\right]
\end{gathered}
$$


TABELA 2- Teste de Esfericidade de Mauchly para as variáveis altura e diâmetro da da planta, de progênies de cupuaçuzeiro,em Tomé-Açu-Pará.

\begin{tabular}{lcccc}
\hline Variáveis & G.L*. & Critério de Mauchly (W) & Aproximação Qui-Quadrado & $\operatorname{Pr}>$ ChiSq \\
\hline Altura & 2 & 0,8267251 & 18,076884 & 0,0001 \\
Diâmetro & 2 & 0,9393412 & 5,9447692 & 0,0512 \\
\hline
\end{tabular}

${ }^{*}$ G.L.: Graus de Liberdade

TABELA3 - Estruturas da matriz de covariâncias, número de parâmetros estimados e Critérios de Informação de Akaike (AIC) e de Schwarz (BIC) no modelo utilizado para a variável altura dda planta, de progênies de cupuaçuzeiro,em Tomé-Açu-Pará.

\begin{tabular}{lccc}
\hline Estruturas da Matriz de Covariâncias & Parâmetros & AIC & BIC \\
\hline Autorregressiva de 1' ${ }^{\mathrm{a}}$ Ordem (AR(1)) & 2 & -122.8 & -105.9 \\
Não Estruturada (UN) & 6 & -96.6 & -90.9 \\
Simetria Composta (CS) & 2 & -123.7 & -112.4 \\
Simetria Composta Heterogênea (CSH) & 4 & -83.8 & -78.1 \\
\hline
\end{tabular}

TABELA 4-Teste de significância dos efeitos principais Progênie e Tempo e da interação Progênie x Tempo para as variáveis altura e diâmetro das plantas de cupuaçuzeiro, em Tomé-Açu-Pará.

\begin{tabular}{ccccccccc}
\hline & \multicolumn{3}{c}{ Variável Altura $^{1}$} & \multicolumn{4}{c}{ Variável Diâmetro $^{2}$} \\
\hline Fonte & G.L. Num. $^{3}$ G.L. Den. & F & Pr $>$ F & G.L. Num. ${ }^{3}$ & G.L. Den. ${ }^{4}$ & F & Pr>F \\
\hline Bloco & 4 & 96 & 2,50 & $0,047^{*}$ & 4 & 96 & 0,9 & $0,478^{\text {ns }}$ \\
Progênie (P) & 24 & 96 & 2,41 & $0,001^{* *}$ & 24 & 96 & 1,4 & $0,113^{\text {ns }}$ \\
Tempo (T) & 2 & 200 & 2093,58 & $0,0^{* *}$ & 2 & 200 & 8092,9 & $0,0^{* *}$ \\
P x T & 48 & 200 & 1,73 & $0,005^{* *}$ & 48 & 200 & 2,3 & $0,0^{* *}$ \\
\hline
\end{tabular}

1: Análise usual em parcelas subdivididas; 2: Estrutura de covariâncias Simetria Composta Heterogênea; 3: Graus de Liberdade do numerador; 4: Graus de Liberdade do denominador; ***: significativo ao nível de 5 e $1 \%$ de probabilidade; ns: não significativo;

TABELA 5 - Altura e Diâmetro da planta, de progênies de cupuaçuzeiro, nos três primeiros de idade, em Tomé-Açu-Pará.

\begin{tabular}{|c|c|c|c|c|c|c|}
\hline \multirow{2}{*}{ Progênies e seus parentais } & \multicolumn{3}{|c|}{ Altura $(\mathrm{cm})^{(1)}$} & \multicolumn{3}{|c|}{ Diâmetro $(\mathrm{cm})^{(1)}$} \\
\hline & 2008 & 2009 & 2010 & 2008 & 2009 & 2010 \\
\hline $117-174 \times(186 \times 434)$ & $1.38 \mathrm{a}$ & $1.83 \mathrm{a}$ & $2.46 \mathrm{~b}$ & $2.38 \mathrm{a}$ & $3.62 \mathrm{a}$ & $6.01 \mathrm{~b}$ \\
\hline $118-174 \times(186 \times 434)$ & $1.61 \mathrm{a}$ & $2.11 \mathrm{a}$ & $2.69 \mathrm{a}$ & $3.24 \mathrm{a}$ & $4.54 \mathrm{a}$ & $6.94 \mathrm{a}$ \\
\hline $120-174 \times(186 \times 434)$ & $1.68 \mathrm{a}$ & $2.11 \mathrm{a}$ & $2.61 \mathrm{a}$ & $2.90 \mathrm{a}$ & $4.12 \mathrm{a}$ & $6.36 \mathrm{~b}$ \\
\hline $121-174 \times(186 \times 434)$ & $1.48 \mathrm{a}$ & $1.99 \mathrm{a}$ & $2.54 \mathrm{a}$ & $2.88 \mathrm{a}$ & $4.13 \mathrm{a}$ & $6.61 \mathrm{~b}$ \\
\hline $122-174 \times(286 \times 215)$ & $1.46 \mathrm{a}$ & $2.02 \mathrm{a}$ & $2.63 \mathrm{a}$ & $2.88 \mathrm{a}$ & $4.26 \mathrm{a}$ & $6.84 \mathrm{a}$ \\
\hline $123-174 \times(186 \times 554)$ & $1.36 \mathrm{a}$ & $1.88 \mathrm{a}$ & $2.48 \mathrm{~b}$ & $2.60 \mathrm{a}$ & $3.98 \mathrm{a}$ & $6.50 \mathrm{~b}$ \\
\hline $124-174 \times(186 \times 554)$ & $1.31 \mathrm{a}$ & $1.78 \mathrm{a}$ & $2.44 \mathrm{~b}$ & $2.24 \mathrm{a}$ & $3.43 \mathrm{a}$ & $6.12 \mathrm{~b}$ \\
\hline $127-174 \times(186 \times 1074)$ & $1.55 \mathrm{a}$ & $2.07 \mathrm{a}$ & $2.66 \mathrm{a}$ & $3.11 \mathrm{a}$ & $4.28 \mathrm{a}$ & $7.17 \mathrm{a}$ \\
\hline $128-174 \times(186 \times 1074)$ & $1.50 \mathrm{a}$ & $2.00 \mathrm{a}$ & $2.39 \mathrm{~b}$ & $2.76 \mathrm{a}$ & $4.04 \mathrm{a}$ & $6.46 \mathrm{~b}$ \\
\hline $129-174 \times(186 \times 1074)$ & $1.64 \mathrm{a}$ & $2.09 \mathrm{a}$ & $2.77 \mathrm{a}$ & $3.00 \mathrm{a}$ & $4.45 \mathrm{a}$ & $7.25 \mathrm{a}$ \\
\hline $131-174 \times(186 \times 513)$ & $1.58 \mathrm{a}$ & $2.05 \mathrm{a}$ & $2.52 \mathrm{a}$ & $2.78 \mathrm{a}$ & $4.22 \mathrm{a}$ & $7.03 \mathrm{a}$ \\
\hline $133-186 \times(286 \times 215)$ & $1.53 \mathrm{a}$ & $2.19 \mathrm{a}$ & $2.77 \mathrm{a}$ & $2.54 \mathrm{a}$ & $4.00 \mathrm{a}$ & $6.56 \mathrm{~b}$ \\
\hline $136-186 \times(174 \times 286)$ & $1.58 \mathrm{a}$ & $2.18 \mathrm{a}$ & $2.67 \mathrm{a}$ & $3.09 \mathrm{a}$ & $4.46 \mathrm{a}$ & $6.87 \mathrm{a}$ \\
\hline $138-215 \times(174 \times 186)$ & $1.58 \mathrm{a}$ & $2.02 \mathrm{a}$ & $2.64 \mathrm{a}$ & $3.06 \mathrm{a}$ & $4.33 \mathrm{a}$ & $6.75 \mathrm{a}$ \\
\hline $139-215 \times(174 \times 186)$ & $1.57 \mathrm{a}$ & $2.07 \mathrm{a}$ & $2.58 \mathrm{a}$ & $2.83 \mathrm{a}$ & $4.19 \mathrm{a}$ & $6.52 \mathrm{~b}$ \\
\hline $140-215 \times(186 \times 434)$ & $1.56 \mathrm{a}$ & $2.04 \mathrm{a}$ & $2.52 \mathrm{a}$ & $2.61 \mathrm{a}$ & $3.98 \mathrm{a}$ & $6.43 \mathrm{~b}$ \\
\hline 147 - SEKÓ x (174 x 186) & $1.41 \mathrm{a}$ & $1.89 \mathrm{a}$ & $2.19 \mathrm{~b}$ & $3.04 \mathrm{a}$ & $4.17 \mathrm{a}$ & $6.39 \mathrm{~b}$ \\
\hline 149 - SEKÓ x (186 x 434) & $1.42 \mathrm{a}$ & $1.83 \mathrm{a}$ & $2.25 \mathrm{~b}$ & $2.85 \mathrm{a}$ & $4.23 \mathrm{a}$ & $6.30 \mathrm{~b}$ \\
\hline $157-554 \times(174 \times 186)$ & $1.50 \mathrm{a}$ & $2.01 \mathrm{a}$ & $2.62 \mathrm{a}$ & $2.60 \mathrm{a}$ & $3.69 \mathrm{a}$ & $6.28 \mathrm{~b}$ \\
\hline $158-1074 \times(174 \times 186)$ & $1.30 \mathrm{a}$ & $1.92 \mathrm{a}$ & $2.53 \mathrm{a}$ & $2.38 \mathrm{a}$ & $3.86 \mathrm{a}$ & $6.25 \mathrm{~b}$ \\
\hline $160-1074 \times(186 \times 434)$ & $1.42 \mathrm{a}$ & $1.98 \mathrm{a}$ & $2.45 \mathrm{~b}$ & $3.00 \mathrm{a}$ & $4.37 \mathrm{a}$ & $6.74 \mathrm{a}$ \\
\hline $161-1074 \times(186 \times 434)$ & $1.32 \mathrm{a}$ & $1.88 \mathrm{a}$ & $2.32 \mathrm{~b}$ & $2.84 \mathrm{a}$ & $4.35 \mathrm{a}$ & $6.31 \mathrm{~b}$ \\
\hline $165-1074 \times(174 \times 286)$ & $1.36 \mathrm{a}$ & $1.86 \mathrm{a}$ & $2.34 \mathrm{~b}$ & $3.07 \mathrm{a}$ & $4.26 \mathrm{a}$ & $6.28 \mathrm{~b}$ \\
\hline $166-1074 \times(186 \times 554)$ & $1.51 \mathrm{a}$ & $1.93 \mathrm{a}$ & $2.28 \mathrm{~b}$ & $2.81 \mathrm{a}$ & $4.08 \mathrm{a}$ & $6.01 \mathrm{~b}$ \\
\hline $167-1074 \times(186 \times 554)$ & $1.38 \mathrm{a}$ & $1.75 \mathrm{a}$ & $2.15 \mathrm{~b}$ & $2.78 \mathrm{a}$ & $4.17 \mathrm{a}$ & $6.54 \mathrm{~b}$ \\
\hline
\end{tabular}




\section{CONCLUSÕES}

Por ser de fácil implementação e por apresentar facilidade na interpretação dos resultados, o esquema de delineamento em parcelas subdivididas no tempo é bastante usado para análise de medidas repetidas. No entanto, aqui também ficou comprovado que essa análise só terá validade se a condição de esfericidade for atendida; caso contrário, deve-se efetuar a análise por meio de técnicas multivariadas ou de modelos mistos.

Para a variável altura da planta, a condição de esfericidade não foi atendida, sendo selecionada a estrutura de covariância residual Simetria Composta Heterogênea.

Diferenças entre as progênies foram encontradas somente no terceiro ano de avaliação, sendo que a variável diâmetro da planta permitiu discriminar melhor as progênies avaliadas.

\section{REFERÊNCIAS}

ALVES, R. M.; RESENDE, M.D. V.D.; BANDEIRA, B. D. S.; PINHEIRO, T. M.; FARIAS, D. C. R. Evolução da vassoura-de-bruxa e avaliação da resistência em progênies de cupuaçuzeiro. Revista Brasileira de Fruticultura, Jaboticabal, v. 31, n.4, p. 1022-1032, 2009.

ALVES, R. M.; RESENDE, M. D. V.D.; BANDEIRA, B. D. S.; PINHEIRO, T. M.; FARIAS, D. C. R. Avaliação e seleção de progênies de cupuaçuzeiro (Theobroma grandiflorum), em Belém, Pará. Revista Brasileira de Fruticultura, Jaboticabal, v. 32, n.1, p. 204-212, 2010.

ALVES, R. M.; SILVA, C. D. S.; SILVA, M. D. C.; SILVA, D. D. S.; SEBBENN, A. M. Diversidade genética em coleções amazônicas de germoplasma de cupuaçuzeiro [Theobroma grandiflorum (Willd. ex Spreng.) Schum.]. Revista Brasileira de Fruticultura, Jaboticabal, v.35, n.3, p. 818-828, 2013.

ALVES, R.M.; FERREIRA, F.N. BRS Carimbó - a nova cultivar de cupuaçuzeiro da Embrapa Amazônia Oriental. Belém: Embrapa Amazônia Oriental, 2012. 8p. (Comunicado Técnico, 232)
AMBROSANO, E. J.; CANTARELLA, H.; AMBROSANO, G. M. B.; SCHAMMAS, E. A.; DIAS, F. L. F., ROSSI, F.; TRIVELIN, P. C. O.; MURAOKA, T.; SACHS, R. C.; AZCON, R. Produtividade da cana-de-açúcar após o cultivo de leguminosas. Bragantia, Campinas, v. 70, p. 810818, 2011.

APARÍCIO, P. D. S.; FERREIRA, R. L. C.; SILVA, J. A. A.; ROSA, A. C.; APARÍCIO, W. C. D. S. Controle da matocompetição em plantios de dois clones de eucalyptus $\times$ urograndis no Amapá. Ciência Florestal, Santa Maria, v.20, p. 381-390, 2010.

CECON, P. R.; SILVA, F. F.; FERREIRA, A.; FERRÃO, R. G.; CARNEIRO, A. P. S.; DETMANN, E.; FARIA, P.N.; MORAIS, T. S. S. Análise de medidas repetidas na avaliação de clones de café 'Conilon'. Pesquisa Agropecuária Brasileira, Brasília, v.43, n.9, p. 1171-1176, 2008.

CESTARI, A. A.; COSTA, S. C.; MINHO, A. P. Análise de dados longitudinais em experimentação animal. Semina: Ciências Agrárias, Londrina,v. 33, p.1565-1580, 2012.

FERREIRA, E. B.; CAVALCANTI, P. P.; NOGUEIRA, D. A. ExpDes: experimental designs package. R package version (1.1.2). 2013. Disponível em: <http://cran.r-project.org/web/packages/ ExpDes/index.html>. Acesso: 26 nov. 2013.

FREITAS, E. G.; BARBIN, D.; BARBOSA, G. V. de S.; CARNEIRO, M. S.; BASSINELLO, A. I. Modelo univariado aplicado a dados longitudinais de cana-de-açúcar. Revista Brasileira de Biometria, São Paulo, v. 26, n.2, p.93-106, 2008.

HUYNH, H.; FELDT, L .S. Condition under which mean square rations in repeated measurements designs have exact F-distributions. Journal of the American Statistical Association, New York, v.72, p.320-40, 1970 .

JELIHOVSCHI, E. G.; FARIA, J. C.; ALLAMAN, I .B. ScottKnott: a package for performing the scottknott clustering algorithm in R. Trends in Applied and Computational Mathematics, São Carlos, v.15, n.1, p.3-17, 2014. Disponível em: $\leq$ http://www. sbmac.org.br/tema/seer/index.php/tema/article/ view/646/643>. 
LYRA, M. R. C. C.; SILVA, J. A. A; ROLIM, M. M. Emprego do teste de esfericidade de Mauchly em um experimento de fertirrigação com vinhaça com medidas repetidas ao longo do tempo. Revista Ciências Agrárias, Belém, n. 45, p. 175-186, 2006.

MAUCHLY, J.W. Significance test for sphericity of a normal n-variate distribuition. Annals of Mathematical Statistics, Ann Arbor, v.11, p.204209, 1940.

MIRANDA, G. V.; SOUZA, L. V.; GUIMARÃES, L. J. M.; NAMORATO, H.; OLIVEIRA, L. R.; SOARES, M. O. Multivariate analyses of genotype $\mathrm{x}$ environment interaction of popcorn. Pesquisa Agropecuária Brasileira, Brasília, v.44, p.45-50, 2009.

OLIVEIRA, T. B.; GENOVESE, M. I. Chemical composition of cupuassu (Theobroma grandiflorum) and cocoa (Theobroma cacao) liquors and their effects on streptozotocin-induced diabetic rats. Food Research International, Barking, v.51, p.929-935, 2013.

PASA, M. S.; FACHINELLO, J. D. S.; FISCHER, D. L. O.; ROSA JÚNIOR, H. F. Performance of rabbiteye and highbush blueberry cultivars as affected by mulching. Revista Brasileira de Fruticultura, Jaboticabal, v. 36, n.1, p. 161-169, 2014.

R Core Team. R: A language and environment for statistical computing. Vienna: R Foundation for Statistical Computing, 2015. Disponível em: $\leq \mathrm{http}: / /$ www.R-project.org/>.
ROSA, R.; TALHAOUI, N.; ROUIS, H.; VELASCO, L.; LEÓN, L. Fruit characteristics and fatty acid composition in advanced olive breeding selections along the ripening period. Food Research International, New York, v. 54, p. 1890-1896, 2013.

SAGRI- Secretária Estadual de Abastecimento e Agricultura. Estatística. Pará, 2015. Disponível em http://www.sagri.pa.gov.br/pagina/agricultura $>$. Acesso em: 15 jul. 2015.

SAS INSTITUTE. SAS user's guide: statistics, version 9.3. Cary: SAS Institute, 2012.

SILVA, E. N.; DUARTE, J. B.; REIS, A. J. Seleção da matriz de variância-covariância residual na análise de ensaios varietais com medidas repetidas em cana-de-açúcar. Ciencia Rural, Santa Maria, v.45, p. 993-999, 2015.

SOUZA, A. das G.C.; SILVA, S.E.L.; SOUZA, N.R. Avaliação de progênies de cupuaçuzeiro (Theobroma grandiflorum (Willd. ex Spreng, Schum) em Manaus. Revista Brasileira de Fruticultura, Jaboticabal, v.20, n.3, p.307-312, 1998.

VONESH, F.E.; CHINCHILLI, V.M. Linear and nonlinear models for the analysis of repeated measurements. New York: Marcel Dekker, 1997. 560p.

XAVIER, L. H., DIAS, C. T. S. Acurácia do modelo univariado para análise de medidas repetidas por simulação multidimensional. Scientia Agricola, Piracicaba, v.58, n.2, p.241-250, 2001. 